\title{
Modeling and Optimization of Electrical Discharge Machining of SiC Parameters, Using Neural Network and Non-dominating Sorting Genetic Algorithm (NSGA II)
}

\author{
Ramezan Ali MahdaviNejad \\ School of mechanical Engineering, College of Engineering, University of Tehran, Tehran, Iran. \\ Email: mahdavin@ut.ac.ir
}

Received December 29 ${ }^{\text {th }}, 2010$; revised March 28 $8^{\text {th }}, 2011$; accepted May $18^{\text {th }}, 2011$.

\begin{abstract}
Silicon Carbide (SiC) machining by traditional methods with regards to its high hardness is not possible. Electro Discharge Machining, among non-traditional machining methods, is used for machining of SiC. The present work is aimed to optimize the surface roughness and material removal rate of electro discharge machining of SiC parameters simultaneously. As the output parameters are conflicting in nature, so there is no single combination of machining parameters, which provides the best machining performance. Artificial neural network (ANN) with back propagation algorithm is used to model the process. A multi-objective optimization method, non-dominating sorting genetic algorithm-II is used to optimize the process. Affects of three important input parameters of process viz., discharge current, pulse on time $\left(T_{\text {on }}\right)$, pulse off time $\left(T_{\text {off }}\right)$ on electric discharge machining of SiC are considered. Experiments have been conducted over a wide range of considered input parameters for training and verification of the model. Testing results demonstrate that the model is suitable for predicting the response parameters. A pareto-optimal set has been predicted in this work.
\end{abstract}

Keywords: Electro Discharge Machining, Non-Dominating Sorting Algorithm, Neural Network, REFEL SiC

\section{Introduction}

Electrical discharge machining (EDM) is one of the most extensively used non-conventional material removal process. Its unique feature of using thermal energy to machine electrically conductive parts regardless of hardness has been its distinctive advantage in the manufacture of mould, die, automotive, aerospace and surgical component [1]. The selection of appropriate parameters for maximum material removal rate and minimum surface roughness during the EDM process traditionally carried out by the operator's experience or conservative technological data provided by the EDM equipment manufacturers, which produced inconsistent machining performance.[2]

Some researchers carried out various investigations to improve the stock material removal rate and surface finishing in EDM process. Proper selection of machining parameters for the best process performance is still a challenging job.

Wang et al. [3] used genetic algorithm (GA) with artificial neural network (ANN) to find out optimal main output parameters such as material removal rate and surface roughness. They used ANN to model the process and Hunter Software to solve multi-objective optimization problem. Using ANN and GA, Su et al. [4] optimized EDM parameters, roughing and finishing machining stages. They utilized artificial neural network to establish the relationship between the process parameters and outputs. GA with properly defined objective functions was then adapted to the neural network to determine the optimal process parameters. They transformed material removal rate, tool wear and surface roughness into a single objective. Rao et al. [7] used ANN and GA to optimize the surface roughness of die sinking electric discharge machining (EDM) by considering the simultaneous affect of various input parameters. Genetic algorithm concept was used to optimize the weighting factors 
of the network.

Pal et al. [1] used non dominating sorting genetic algorithm-II to optimize the process. They conducted some experiments on $\mathrm{C} 40$ Steel to generate input and output data for training an ANN model. Material removal rate and tool wear were two objectives to be optimized. So they predicted a pareto-optimal set for outputs.

In this study material removal rate and surface roughness have been considered to produce a pareto-optimal set for EDM of REFEL SiC. Some related properties of this material are shown in Table 1.

\section{Experimentations}

In this study, Deckel CNC Spark, ISO frequency system, with gap control system was used to carry out the experiments. Copper electrode was selected to drill holes in the REFEL SiC blocks. For evaluating the EDM process the MRR and surface roughness (Ra) are mentioned with input machining parameters such as pulse on time $\left(T_{o n}\right)$, pulse off time $\left(T_{\text {off }}\right)$, discharge current $(I)$. Proper selection of the machining parameters can result a higher material removal rate and lower Ra. Using an orthogonal array $L_{25}$ according Taguchi method decreased the number experiments effectively. Hence 25 sets of experiments have been conducted with five levels of each parameter (current, pulse on time and off time) to collect for training of the neural network model. Moreover five sets of experiments have been for testing the trained neural network. For each experiment, a new set of tool and work-piece has been used. For normal polarity the work-piece is connected to the negative terminal and the tool is connected to the positive terminal of the source, where as for reverse polarity it is just the opposite. Experiment has been performed with normal polarity. The current range is $0.1-5 \mathrm{~A}$ and the pulse on time and pulse off time ranges are $21-1125 \mu \mathrm{s}$.

\section{Material Removal Rate (MRR)}

Material removal rate and surface roughness have been used to evaluate machining performance. Material removal rate (MRR) is calculated from the difference of weight of work piece before and after experiment.

$$
\operatorname{MRR}=\frac{\left(w_{i}-w_{f}\right)}{\rho_{\mathrm{SiC}} t} \mathrm{~mm}^{3} / \mathrm{min}
$$

where, $w_{i}$ is the initial weight of workpiece in $\mathrm{g} ; w_{f}$ the weight of workpiece after machining in $g$; $t$ the machining time in minutes; $\rho_{\mathrm{SiC}}$ is the density of $\mathrm{SiC}(3.1 \times$ $10^{-3} \mathrm{~g} / \mathrm{mm}^{3}$ ).

\section{Surface Roughness}

The surface roughness $R_{a}$ is the arithmetic average of collected roughness data points and given by the sum of the absolute values of all the areas above and below the mean line (in integrally form). A mean line is found that is parallel to the general surface direction and divides the surface in such a way that the sum of the areas formed above the line is equal to the sum of the areas formed below the line. When sample points were taken, $R_{a}$ is calculated as follows:

$$
R_{a}=\frac{1}{n} \sum_{i=1}^{n}\left|y_{i}\right|
$$

where $y_{i}$ is the distance between the $i^{\text {th }}$ sample point on the profile from the mean line, and $\mathrm{n}$ is the number of sample points.

\section{Neural Network}

Modeling of EDM with feed forward neural network is composed of two stages: training and testing of the network with experimental machining data. The scale of the input and output data is an important matter to consider, especially, when the operating ranges of process parameters are different. The scaling or normalization ensures that the ANN will be trained effectively. By searching in different network architectures using a MATLAB code, multilayer-perceptron (3-5-5-2) was chosen as the network architecture. The networks were trained using a back-propagation algorithm. The selected network architecture had the minimum value of the error. The error $E$ indicates the difference between the actual and the desired output of the neural network, as follows:

$$
E=\sum_{j=1}^{a z}\left(y_{j}-a_{j}\right)^{2} \rightarrow \min
$$

where $y_{j}$ is the desired output, $a_{j}$ is the calculated output, $a z$ the number of testing data. Five sets of experiments allocated to test the network's error value. Pulse on-time, pulse off-time and the current are the inputs of neural network and material removal rate and surface roughness

\begin{tabular}{|c|c|c|c|c|c|c|c|c|}
\hline $\begin{array}{l}\text { Density } \\
\left(\mathrm{gr} / \mathrm{cm}^{3}\right)\end{array}$ & $\begin{array}{l}\text { Hardness } \\
\text { (HV) }\end{array}$ & $\begin{array}{l}\text { Young modulus } \\
\text { (E) }(\mathrm{GN} / \mathrm{m})\end{array}$ & $\begin{array}{c}\text { Thermal } \\
\text { expansion } \\
1 \times 10^{-60} \mathrm{C}\end{array}$ & $\begin{array}{r}\text { Thermal } \\
\text { (k) at } 100 \\
\text { at } 1\end{array}$ & $\begin{array}{l}\text { aductivity } \\
\left(\mathbf{W} / \mathbf{m} \cdot{ }^{\circ} \mathbf{C}\right) \\
\mathbf{0}^{\circ} \mathbf{C}\end{array}$ & $\begin{array}{c}\text { Specific heat } \\
\left(\mathbf{J} / \mathbf{g}^{\circ} \mathbf{C}\right)\end{array}$ & $\begin{array}{c}\text { Electrical resistance } \\
(\Omega \cdot \mathrm{cm})\end{array}$ & $\begin{array}{l}\text { Thermal shock } \\
(\mathrm{cal} / \mathrm{cm} \cdot \mathrm{s}) \text { at } 500^{\circ} \mathrm{C}\end{array}$ \\
\hline 3.10 & 2500 & 413 & 4.3 & 83.6 & 38.9 & 670.710 & $\begin{array}{c}0.42\left(\text { at } 25^{\circ} \mathbf{C}\right) \\
0.016\left(\text { at } 1200^{\circ} \mathbf{C}\right)\end{array}$ & 59 \\
\hline
\end{tabular}
are the outputs of the neural network. Figure 1 shows the

Table1. Some characteristics of REFEL SiC [5]. 
material removal rate comparison between experimental outputs and the corresponding values that are predicted by neural network. The average percentage of error for predicting MRR is $6.71 \%$.

Figure 2 shows the surface roughness comparison between experimental outputs and the corresponding values that are predicted by neural network. The average percentage of error for predicting is $5.67 \%$ in this case.

\section{NSGA II}

A single objective optimization algorithm provides a single optimal solution. However, most of the multi-objective problems, in principle, give rise to a set of optimal solutions instead of a single optimal solution [1-9]. The set of solution is known as pareto-optimal solution. In the absence of any further information, none of these pareto-optimal solutions cannot be said to be better than the other. Suitability of one solution depends on a number of factors including user's choice and problem environment and etc. Hence, this demands finding the entire set of optimal solutions. In this study two objectives that we considered are MRR and Ra. It is observed that when MRR is increasing the Ra increases too. But our goals are maximizing of MRR and minimizing of Ra. A single optimal solution will not serve our purpose, as these objectives are conflicting in nature. Optimization of both the output parameters requires multi-objective optimization. Genetic algorithm works with a population of feasible solutions and, therefore, it can be used in multi-objective optimization problems to capture a number of solutions simultaneously. NSGA-II is fast and elitist multi objective GA, proposed by Dev et al. [6]. The flow chart of NSGA-II is shown in Figure 3.

\section{Discussion}

The objectives in this study, which are conflicting together, are MRR and surface roughness. In order to convert the first objective (MRR) for minimization, it is suitably modified. Two objective functions are given below:

$$
\text { objective } 1=1 / \text { MRR and objective } 2=R_{a}
$$

The non-dominated solution set obtained over the entire optimization procedure is shown in Figure 4. This shows the formation of the pareto-optimal front leading to the final set of solutions.

Since none of the solutions in the pareto-optimal front is absolutely better than any other, any one of them is an acceptable solution. The choice of one solution over the other depends on the requirement of the process engineer. If the situation or environment can permit a surface roughness rate of $3 \mu \mathrm{m}$ to maintain the accuracy of the

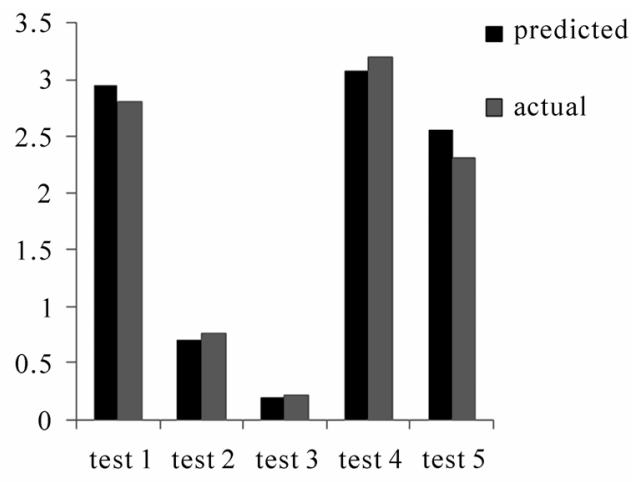

Figure 1. Comparison between experimental and neural network predicted outputs of material removal rate.

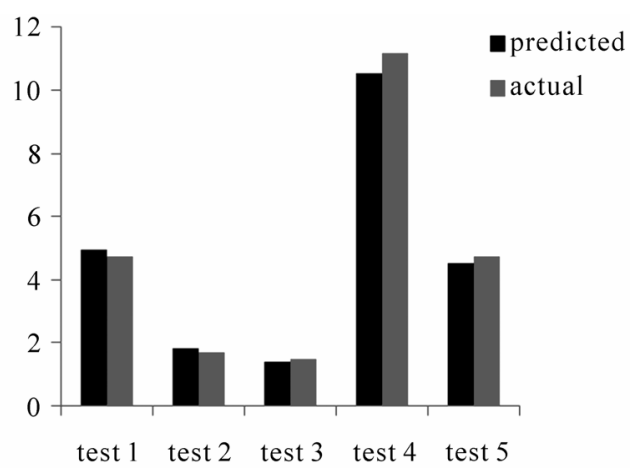

Figure 2. Comparison between experimental and neural network predicted outputs of surface roughness.

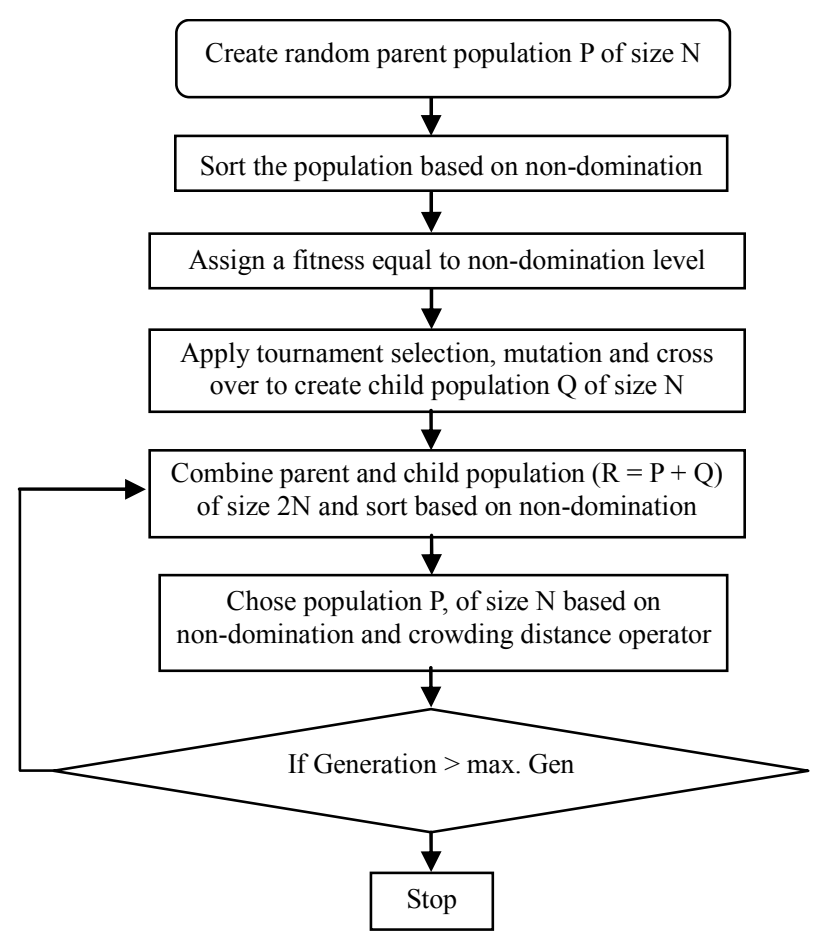

Figure 3. Flow chart of NSGA II. 

Non-dominating Sorting Genetic Algorithm (NSGA II)

Table 2. Optimal sets of parameters.

\begin{tabular}{|c|c|c|c|c|c|}
\hline & $\mathbf{T i}$ & To & I & MRR & $\mathbf{R a}$ \\
\hline 1 & 858.9584 & 924.4639 & 4.8902 & 3.6446 & 8.4364 \\
\hline 2 & 141.5017 & 496.6855 & 0.1 & 0.3466 & 0.886 \\
\hline 3 & 174.6221 & 682.9052 & 1.0473 & 1.4528 & 1.1667 \\
\hline 4 & 316.126 & 21 & 3.8979 & 1.9901 & 1.1703 \\
\hline 5 & 206.7583 & 663.0539 & 4.1795 & 3.5945 & 5.5242 \\
\hline 6 & 168.7212 & 536.2421 & 4.2317 & 3.5695 & 5.0381 \\
\hline 7 & 429.4838 & 70.5361 & 4.5337 & 2.3535 & 1.4294 \\
\hline 8 & 407.6555 & 101.8867 & 4.5579 & 2.4078 & 1.5658 \\
\hline 9 & 431.1151 & 33.2454 & 4.5007 & 2.2597 & 1.2826 \\
\hline 10 & 372.2205 & 1082.09 & 3.9459 & 2.5234 & 1.6222 \\
\hline 11 & 299.4541 & 937.1161 & 3.2485 & 3.2904 & 3.5347 \\
\hline 12 & 349.6336 & 21 & 4.2386 & 2.1084 & 1.1764 \\
\hline 13 & 133.5872 & 406.38 & 3.9202 & 3.4407 & 4.251 \\
\hline 14 & 180.275 & 545.7843 & 0.5586 & 0.676 & 0.9604 \\
\hline 15 & 120.2597 & 409.5408 & 3.9015 & 3.4017 & 4.0785 \\
\hline 16 & 942.8562 & 855.8315 & 4.5168 & 3.6315 & 6.7868 \\
\hline 17 & 287.1614 & 1024.018 & 3.0806 & 2.8177 & 1.7393 \\
\hline 18 & 284.3813 & 1032.874 & 3.0924 & 2.7484 & 1.7079 \\
\hline 19 & 877.9769 & 938.2853 & 4.871 & 3.6442 & 7.9565 \\
\hline 20 & 171.6979 & 605.3086 & 0.7507 & 0.9007 & 1.0196 \\
\hline 21 & 194.7966 & 529.194 & 0.7099 & 0.8277 & 1.0017 \\
\hline 22 & 403.2268 & 21.0838 & 4.4904 & 2.1987 & 1.2137 \\
\hline 23 & 300.4237 & 948.3546 & 3.2485 & 3.2873 & 3.117 \\
\hline 24 & 113.1908 & 369.4054 & 3.8439 & 3.2937 & 3.8086 \\
\hline 25 & 917.4061 & 941.7156 & 4.8294 & 3.6407 & 7.5638 \\
\hline 26 & 916.4971 & 891.0667 & 4.6921 & 3.6398 & 7.379 \\
\hline 27 & 984.2048 & 816.5333 & 4.4308 & 3.6187 & 6.2749 \\
\hline 28 & 303.9082 & 938.2599 & 3.2566 & 3.2897 & 3.3114 \\
\hline 29 & 1046.005 & 805.3527 & 4.3747 & 3.5948 & 5.7939 \\
\hline 30 & 870.1822 & 924.2211 & 4.8671 & 3.6445 & 8.21 \\
\hline 31 & 281.8689 & 1032.299 & 3.0245 & 2.6488 & 1.6937 \\
\hline 32 & 315.6009 & 21 & 3.9119 & 1.993 & 1.1704 \\
\hline 33 & 181.5296 & 531.0391 & 0.6132 & 0.7553 & 0.9725 \\
\hline 34 & 275.9709 & 1016.142 & 3.0625 & 2.9787 & 1.809 \\
\hline 35 & 131.772 & 491.3254 & 4.1534 & 3.5081 & 4.5261 \\
\hline 36 & 305.2944 & 948.2171 & 3.2467 & 3.2833 & 2.8871 \\
\hline 37 & 861.9278 & 930.9182 & 4.8708 & 3.6445 & 8.1551 \\
\hline
\end{tabular}



Non-dominating Sorting Genetic Algorithm (NSGA II)

\begin{tabular}{|c|c|c|c|c|c|}
\hline 38 & 119.1833 & 477.8889 & 4.0229 & 3.4609 & 4.3141 \\
\hline 39 & 376.0995 & 24.0189 & 4.3534 & 2.1632 & 1.1971 \\
\hline 40 & 173.1123 & 685.8219 & 1.0394 & 1.4358 & 1.1631 \\
\hline 41 & 284.2434 & 1035.765 & 3.0867 & 2.6966 & 1.6946 \\
\hline 42 & 286.2831 & 1021.49 & 3.0881 & 2.8707 & 1.7557 \\
\hline 43 & 148.4211 & 482.6119 & 0.1 & 0.3784 & 0.886 \\
\hline 44 & 298.755 & 937.8997 & 3.1764 & 3.2873 & 3.0139 \\
\hline 45 & 308.5639 & 948.8102 & 3.1985 & 3.2631 & 2.5244 \\
\hline 46 & 366.4247 & 1078.362 & 3.9262 & 2.593 & 1.6522 \\
\hline 47 & 996.3369 & 816.5368 & 4.4229 & 3.6152 & 6.1633 \\
\hline 48 & 115.4423 & 368.0682 & 3.9275 & 3.3269 & 3.8915 \\
\hline 49 & 168.5636 & 634.8079 & 0.9029 & 1.183 & 1.095 \\
\hline 50 & 176.8979 & 645.9134 & 0.9506 & 1.2511 & 1.1102 \\
\hline 51 & 172.9696 & 685.0409 & 1.0058 & 1.3451 & 1.1468 \\
\hline 52 & 304.4127 & 946.2207 & 3.2116 & 3.2803 & 2.772 \\
\hline 53 & 157.1823 & 527.6218 & 4.3731 & 3.5523 & 4.8573 \\
\hline 54 & 925.4805 & 861.5791 & 4.5397 & 3.6341 & 6.9647 \\
\hline 55 & 149.998 & 493.2173 & 4.2715 & 3.5425 & 4.7609 \\
\hline 56 & 306.9045 & 981.8667 & 3.249 & 3.2036 & 2.1755 \\
\hline 57 & 302.2196 & 938.9002 & 3.2589 & 3.2901 & 3.3997 \\
\hline 58 & 908.0573 & 923.543 & 4.8141 & 3.6424 & 7.6703 \\
\hline 59 & 927.5109 & 896.1882 & 4.6304 & 3.6355 & 7.13 \\
\hline 60 & 113.3054 & 371.2426 & 4.1168 & 3.36 & 4.0045 \\
\hline 61 & 163.5354 & 448.252 & 0.1 & 0.4499 & 0.8898 \\
\hline 62 & 161.4533 & 546.5079 & 4.2317 & 3.5642 & 4.9386 \\
\hline 63 & 175.078 & 447.2047 & 0.3503 & 0.5919 & 0.9336 \\
\hline 64 & 190.6357 & 418.0032 & 0.4155 & 0.6059 & 0.9571 \\
\hline 65 & 184.3604 & 585.3931 & 0.7912 & 0.9543 & 1.0309 \\
\hline 66 & 968.7002 & 849.109 & 4.5269 & 3.6279 & 6.6203 \\
\hline 67 & 138.972 & 500.3413 & 4.1505 & 3.5256 & 4.6216 \\
\hline 68 & 179.8468 & 438.7951 & 0.2235 & 0.5309 & 0.9089 \\
\hline 69 & 305.3556 & 971.6719 & 3.2454 & 3.2423 & 2.3567 \\
\hline 70 & 285.3319 & 1012.133 & 3.0527 & 2.9273 & 1.7853 \\
\hline 71 & 133.0257 & 431.1921 & 4.0818 & 3.4829 & 4.3986 \\
\hline 72 & 959.5319 & 825.0148 & 4.421 & 3.6244 & 6.4444 \\
\hline 73 & 183.5624 & 532.4206 & 0.6413 & 0.7817 & 0.9806 \\
\hline 74 & 368.9871 & 1078.629 & 3.9218 & 2.5689 & 1.6401 \\
\hline 75 & 1009.683 & 804.5483 & 4.4012 & 3.6083 & 6.0169 \\
\hline
\end{tabular}



Non-dominating Sorting Genetic Algorithm (NSGA II)

\begin{tabular}{|c|c|c|c|c|c|}
\hline 76 & 176.643 & 618.4106 & 0.9651 & 1.2652 & 1.1409 \\
\hline 77 & 134.2186 & 431.9405 & 4.1533 & 3.4907 & 4.4421 \\
\hline 78 & 961.3217 & 825.2114 & 4.4227 & 3.6242 & 6.4331 \\
\hline 79 & 172.7149 & 683.4143 & 0.9945 & 1.3194 & 1.1413 \\
\hline 80 & 301.2341 & 945.2716 & 3.1541 & 3.2729 & 2.6102 \\
\hline 81 & 300.4964 & 945.9148 & 3.165 & 3.2768 & 2.6725 \\
\hline 82 & 143.7192 & 487.6615 & 4.2564 & 3.5319 & 4.6777 \\
\hline 83 & 174.965 & 613.1224 & 0.8278 & 1.03 & 1.05 \\
\hline 84 & 159.3381 & 649.6514 & 0.8593 & 1.0943 & 1.0768 \\
\hline 85 & 966.0411 & 847.5901 & 4.5269 & 3.6284 & 6.6415 \\
\hline 86 & 305.7388 & 981.1644 & 3.2702 & 3.2229 & 2.2694 \\
\hline 87 & 925.6356 & 861.3723 & 4.556 & 3.6351 & 7.005 \\
\hline 88 & 276.5219 & 994.4977 & 2.9962 & 3.0797 & 1.8856 \\
\hline 89 & 886.8913 & 930.2199 & 4.8374 & 3.6436 & 7.8378 \\
\hline 90 & 182.7754 & 581.0766 & 0.8067 & 0.9855 & 1.0385 \\
\hline 91 & 988.0573 & 799.9433 & 4.3565 & 3.6126 & 6.0526 \\
\hline 92 & 293.829 & 1021.771 & 3.1564 & 2.9067 & 1.7773 \\
\hline 93 & 178.411 & 630.8943 & 0.905 & 1.1575 & 1.0854 \\
\hline 94 & 298.351 & 964.5621 & 3.0682 & 3.1826 & 2.0974 \\
\hline 95 & 1001.796 & 801.5891 & 4.3056 & 3.605 & 5.8814 \\
\hline 96 & 158.8948 & 650.0343 & 0.8593 & 1.0945 & 1.0774 \\
\hline 97 & 278.2378 & 991.8914 & 2.9667 & 3.0496 & 1.8586 \\
\hline 98 & 283.6434 & 966.2962 & 3.093 & 3.253 & 2.4188 \\
\hline 99 & 935.8522 & 898.663 & 4.6975 & 3.6379 & 7.2202 \\
\hline 100 & 115.3983 & 370.507 & 3.8796 & 3.3179 & 3.8653 \\
\hline
\end{tabular}

Scatterplot of Ra Vvs MRR

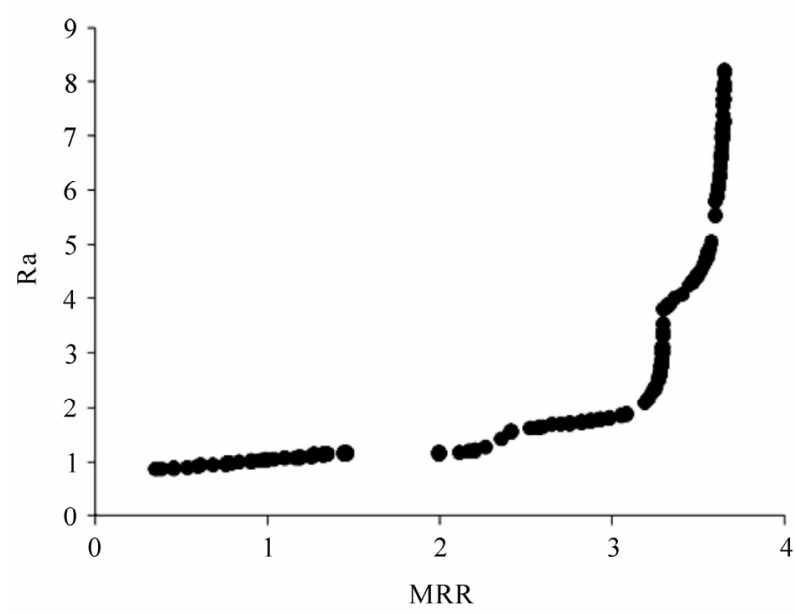

Figure 4. Pareto-optimal set. product, the process engineer can chose the parameter setting according to that to obtain maximum material removal rate at the specified value of surface roughness.

From the experiments results, material removal and surface roughness are $3.58 \mathrm{~mm}^{3} / \mathrm{min}$ and $7.34 \mu \mathrm{m}$ respectively. In this case the pulse on and pulse off times and also the current settings are $850 \mu \mathrm{s}, 900 \mu \mathrm{s}$ and $5 \mathrm{~A}$ respectively. For solution number 1 in Figure 1, material removal rate and Surface roughness are $3.6446 \mathrm{~mm}^{3} / \mathrm{min}$ and $7.2561 \mu \mathrm{m}$, where the pulse on and pulse off times and also current settings are 858.9584, $924.463 \mu$ s and 4.8902A, respectively. Choice of pulse on time and off time will help to achieve higher MRR with same tool wear. This indicates, values obtained from the optimization technique are in close agreement with the experimental values for more or less the same parameter settings. 


\section{Conclusions}

81 experiments have been conducted with a wide range of current, pulse on time and pulse off time. The MRR and surface roughness have been measured for each setting of pulse on time and pulse off time and current. An ANN model has been trained within the experimental data. Various ANN architectures have been studied, and 3-5-5-2 is selected. Material removal rate and surface roughness have been optimized as objectives by using a multi-objective optimization method. Non-dominating sorting genetic algorithm-II and finally pareto-optimal sets of material removal rate and surface roughness are obtained. The results are shown in Table 2.

\section{REFERENCES}

[1] S. K. Pal, D. Mandal and P. Saha, "Modeling of Electrical Discharge Machining Process Using Back Propagation Neural Network and Multi-Objective Optimization Using Non-Dominating Sorting Genetic Algorithm-II," Journal of Materials Processing Technology, Vol. 186, No. 1-3, 2007, pp. 154-162. doi:10.1016/j.jmatprotec.2006.12.030

[2] G. K. M. Rao, G. Rangajanardhaa, D. H. Rao and M. S. Rao, "Development of Hybrid Model and Optimization of Surface Roughness in Electric Discharge Machining Using Artificial Neural Networks and Genetic Algorithm," Journal of Materials Processing Technology, Vol. 209,
No. 3, 2009, pp. 1512-1520.

doi:10.1016/j.jmatprotec.2008.04.003

[3] K. Wang, H. L. Gelgele, Y. Wang, Q. Yuan and M. Fang, "A Hybrid Intelligent Method for Modeling the EDM Process," International Journal of Machine Tools and Manufacture, Vol. 43, No. 10, 2003, pp. 995-999. doi:10.1016/S0890-6955(03)00102-0

[4] J. C. Su, J. Y. Kao and Y. S. Tarng, "Optimization of the Electrical Discharge Machining Process Using a GABased Neural Network," International Journal of Advanced Manufacturing Technology, Vol. 24, 2004, pp. 81-90.

[5] R. Mahdavinejad, M. Tolouei-Rad and H. Sharifi Bidgoli, "Heat Transfer Analysis of EDM Process on Silicon Carbide," International Journal of Numerical Methods for Heat and Fluid Flow, Vol. 15, No. 5, 2005, pp. 483-502. doi:10.1108/09615530510593657

[6] K. Dev, A. Pratap, S. Agarwal and T. Meyarivan, "A Fast and Elitist Multi Objective Genetic Algorithm: NSGA-II," IEEE Transactions on Evolutionary Computation, Vol. 6, No. 2, 2002, pp. 182-197.

[7] G. K. M. Rao, G. Rangajanardhaa, D. H. Rao and M. S. Rao, "Development of Hybrid Model and Optimization of Surface Roughness in Electric Discharge Machining Using Artificial Neural Networks and Genetic Algorithm," Journal of Materials Processing Technology, Vol. 209, No. 3, 2009, pp. 1512-1520. doi:10.1016/j.jmatprotec.2008.04.003 